Audiology

Neurotology
Audiol Neurotol 2010;15:261-272

DOI: $\underline{10.1159 / 000258682}$
Received: January 21, 2009

Accepted after revision: September 28, 2009

Published online: November 17, 2009

\title{
Differentiating Malingering Balance Disorder Patients from Healthy Controls, Compensated Unilateral Vestibular Loss, and Whiplash Patients Using Stance and Gait Posturography
}

\author{
Jaap Vonk Corinne G.C. Horlings John H.J. Allum \\ Division of Audiology and Neurootology, University Hospital, Basel, Switzerland
}

\section{Key Words}

Malingering balance disorder • Unilateral vestibular loss •

Whiplash patients $\cdot$ Stance and gait posturography

\begin{abstract}
Differentiating balance disorder patients who are malingering from those with organic balance disorders is difficult and costly. We used trunk sway measured during several stance and gait tasks in 18 patients suspected of malingering in order to differentiate these from 20 patients who had suffered unilateral vestibular loss 3 months earlier, 20 patients with documented whiplash injuries, and 34 healthy controls. Classification results ranged from 72 to $96 \%$ and were equally accurate for task or criteria variables based on $90 \%$ sway values. The tasks yielding the best discrimination were: standing with eyes closed on a foam and firm surface; standing with eyes open on a firm surface; standing on 1 leg; and walking tandem steps. The criteria yielding the best discrimination were: standing with eyes open on a firm surface; the difference between standing with eyes closed on foam and firm surfaces; the difference between walking tandem steps and standing on 1 leg with eyes open; and the difference between roll and pitch velocity when walking 8 tandem steps. We conclude that discriminating suspected malingering balance disorder patients is possible using variables or criteria based on objective measures of trunk sway during several stance and gait tasks.

Copyright $\odot 2009$ S. Karger AG, Basel
\end{abstract}

\section{Introduction}

When a patient complains of balance problems, the examining physician is faced with the task of assessing the patient's problems and associating these with a corresponding diagnosis. In addition to organic balance disorders such as those associated with a unilateral peripheral vestibular loss (UVL), patients with non-organic aetiologies form a large majority of patients presenting with balance problems. Non-organic vertigo has been cited as the most common cause of the subjective symptoms of vertigo [Nedzelski et al., 1986] with some forms improving under therapy [Querner et al., 2000]. Among those within the non-organic spectrum, 'malingering' patients are those deliberately fabricating or aggravating a balance deficit with the goal of achieving a financial benefit from their 'disorder' which is almost always 'connected' with an automobile accident. The incentive for financial gain differs across Europe, depending on the local legal requirements to prove incapacitating balance problems after an automobile accident [Committee of European Assurances, 2004]. Thus it is important to judge whether patients with apparently debilitating balance problems

Conflict of Interest: The authors state that J.H.J. Allum has worked as a consultant for the company producing the equipment used in this study.

\section{KARGER}

Fax +4161306 1234 E-Mail karger@karger.ch www.karger.com

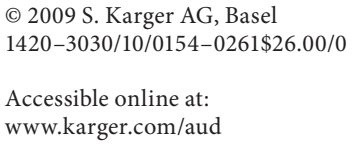

Prof. Dr. J.H.J. Allum

Department of Audiology and Neurootology, Universitätsspital Basel

CH-4031 Basel (Switzerland)

Tel. +41 61265 2040, Fax +41612652750

E-Mail jallum@uhbs.ch 
are consciously mimicking a balance deficit, especially when there is a possibility of financial gain [Binder and Rohling, 1996; Gianoli et al., 2000].

Distinguishing organic and malingering symptoms is difficult because traditional vestibular function tests, such as the caloric test and rotating chair test, normally only provide information on the functional status of the peripheral horizontal circular canal system and the vestibular ocular reflex [Hain, 1995]. Other standard clinical tests, examining vestibular-spinal function, exist but are limited to the neck musculature [Colebatch et al., 1994]. One aspect of vestibular ocular reflex tests on patients with a possible organic base to their balance problems is that they may aggravate their symptoms, worsening the clinical picture, by failing to cooperate and follow instructions. This complicates the differentiation of patients who are malingering from those with organic symptoms.

Several techniques have been suggested to identify malingering patients. One of these, dynamic posturography, has the advantage that vestibular and proprioceptive contributions to balance control can be measured if a toe-up tilt of the support surface is used [for a review, see Allum and Shepard, 1999; Allum et al., 2001]. Thus it has been argued that tilt responses of dynamic posturography can be used to determine objectively whether someone is consciously influencing their balance performance [Allum et al., 1996]. Another form of dynamic posturography, as manufactured by Neurocom ${ }^{\mathrm{TM}}$ (www.equitest.org), uses a sway-referenced support surface (i.e. one that moves with centre of foot pressure variations). The malingering or aphysiologic pattern for this system was initially described by Hamid [1990]. This qualitative description used the following criteria: an abnormally high sway score on simple test conditions, significant intertrial changes within the same tasks, voluntary control of posture, and finally a relatively better balance when being confronted with more difficult circumstances. Cevette et al. [1995] tested 3 different patient groups using the same equipment: patients with aphysiologic patterns who demonstrated unusual symptoms of imbalance, gait unsteadiness, or vertigo; age-matched patients with vestibular dysfunction patterns; and healthy age-matched patients with normal patterns. They established, based on a discriminant analysis, 3 mathematical formulas which differentiated between these 3 groups. These 3 formulas were based on: standing on 2 legs with eyes open on a firm support, standing on 2 legs with eyes open on the sway-referenced support surface, and standing on 2 legs with visual surround and support surface sway-referenced together.
These formulas obtained a noteworthy overall classification rate of $95.5 \%$ between the 3 study groups. Rey-Martinez et al. [2007] applied 7 criteria based on sway-referenced dynamic posturography, including the 3 formulas determined by Cevette et al. [1995], to a patient group of 7 malingerers. This group complained about different degrees of instability, but after follow-up examinations were classified as malingerers. The 3 formulas of Cevette et al. [1995] produced the best results; $88 \%$ of the malingerers were correctly classified. Mallinson et al. [2005] developed a new set of criteria to identify malingering patients which included those of Cevette et al. [1995], Gianoli et al. [2000], and Goebel et al. [1997]. The new criteria which differed were: scoring a patient's performance when the patient is unaware of being assessed, a rhythmic sway pattern throughout all tasks, and finally exaggerated or erratic responses which could not be reproduced. Mallinson and Longridge [2005] were able to detect all 3 malingering patients, who were diagnosed as malingerers based on clinical tests, out of their total research group of 109 patients with balance and/or dizziness complaints. Other scoring systems (those of Cevette et al. [1995] and Gianoli et al. [2000]) detected the 3 malingerers as well, but also raised suspicions in patients who were not originally assessed in clinical tests as being malingerers.

Thus despite some doubts [see Morgan et al., 2002], sway-referenced dynamic posturography appears to be able to objectively determine a malingerer's balance performance. Nonetheless, this technique has some disadvantages. First of all, it only uses values obtained during normal and sway-referenced stance tasks, whereas many patients with balance problems complain of instability during walking. Secondly, sway-referencing tasks can be performed more cheaply on a foam support surface [Weber and Cass, 1993; Allum et al., 2002]. Thirdly, for adequate identification of a patient's instability due to proprioceptive loss during stance, roll (sideways) sway measures are required [Horlings et al., 2008]. Sway-referenced dynamic posturography is restricted to measures in the pitch (foreaft) direction which is adequate to identify vestibular loss [Allum and Adkin, 2003; Horlings et al., 2008].

In this study, a portable measurement system (Swaystar $^{\mathrm{TM}}$ ) attached to the trunk was used to measure trunk sway in the pitch and roll directions during several stance and gait tasks, in order to differentiate patients who are suspected of malingering from healthy controls and patients whose unilateral peripheral vestibular loss was in the 3-month stage of compensation. We also included whiplash injury patients who also suffer from specific balance problems [Sjöström et al., 2003] and are difficult 
Table 1. Malingering patient characteristics

\begin{tabular}{|c|c|c|c|c|c|c|c|c|c|c|}
\hline Subject & Age & Sex & $\mathrm{CP}$ & $20 \mathrm{RCA}$ & $\mathrm{OKN}$ & ETT & Saccades & VEMPs & Accident & $\begin{array}{l}\text { Skull fracture } \\
\text { MRI/CT }\end{array}$ \\
\hline 1 & 46 & $\mathrm{~m}$ & normal & normal & normal & normal & normal & normal & present & no fracture \\
\hline 2 & 30 & $\mathrm{w}$ & path. & path. & path. & normal & normal & path. & present & no fracture \\
\hline 3 & 46 & $\mathrm{w}$ & normal & normal & normal & normal & normal & normal & present & possible fracture \\
\hline 4 & 32 & $\mathrm{w}$ & \# & \# & \# & \# & normal & normal & present & no fracture \\
\hline 5 & 40 & $\mathrm{~m}$ & normal & normal & normal & normal & normal & * & present & no fracture \\
\hline 6 & 48 & $\mathrm{~m}$ & normal & normal & normal & normal & normal & normal & not present & \# \\
\hline 7 & 48 & $\mathrm{~m}$ & normal & normal & normal & normal & normal & normal & present & no fracture \\
\hline 8 & 46 & $\mathrm{w}$ & * & normal & * & * & normal & * & not present & no fracture \\
\hline 9 & 43 & $\mathrm{~m}$ & normal & normal & normal & normal & normal & normal & present & no fracture \\
\hline 10 & 49 & $\mathrm{~m}$ & path. & normal & \# & \# & \# & normal & present & no fracture \\
\hline 11 & 48 & $\mathrm{w}$ & & normal & normal & normal & normal & normal & not present & no fracture \\
\hline 12 & 30 & $\mathrm{~m}$ & normal & normal & normal & normal & normal & normal & present & no fracture \\
\hline 13 & 42 & $\mathrm{~m}$ & \# & normal & \# & \# & \# & \# & present & * \\
\hline 14 & 59 & $\mathrm{w}$ & * & * & * & $*$ & * & normal & not present & * \\
\hline 15 & 47 & $\mathrm{~m}$ & normal & normal & \# & \# & normal & $*$ & not present & no fracture \\
\hline 16 & 38 & $\mathrm{~m}$ & normal & normal & $\#$ & $\#$ & \# & normal & present & no fracture \\
\hline 17 & 53 & $\mathrm{~m}$ & normal & normal & normal & normal & normal & * & present & no fracture \\
\hline 18 & 53 & $\mathrm{w}$ & path. & normal & normal & normal & normal & normal & not present & no fracture \\
\hline
\end{tabular}

$\mathrm{CP}=$ Canal paresis (pathological $>30 \%$ ); $20 \mathrm{RCA}=20 \% \mathrm{~s}^{2}$ rotating chair asymmetry (pathological $>30 \%$, normal with $2 \mathrm{SD}$ of normal range); OKN = optokinetic nystagmus; ETT = eye tracking test; VEMPs = vestibular evoked myogenic potentials; path. = pathological (borderline or outside normal range); ${ }^{*}=$ not tested because of a medical/technical reason; \# = could not test (patient anxiety as a cause or lack of cooperation).

to diagnose [Pearce, 1999]. Thus our main goal was to obtain a selection of balance parameters and criteria to objectively differentiate between malingering patients and those with a UVL; between malingerers and patients with whiplash-associated balance deficits; and between malingerers and normal control subjects using measures of trunk sway. Our secondary goal was to determine if the same criteria could also be used efficiently to separate malingerers from the other populations in one, rather than several separate discriminations. Previous studies have examined differences between UVL patients and controls and between patients with whiplash and controls [Allum and Adkin, 2003; Sjöström et al., 2003; Johansson et al., 1994; Karlberg et al., 1996]. Thus, we have not readdressed these differences here.

\section{Materials and Methods}

\section{Participants}

Eighteen patients who demonstrated unusual symptoms of imbalance, gait unsteadiness, or vertigo and who were diagnosed as suspected malingering patients (mean age \pm SEM, $44 \pm 1.9$ years) were compared with 34 age-matched healthy subjects (46 \pm 1.5 years), 20 age-matched UVL patients 3 months after onset of their acute symptoms ( $54 \pm 3.1$ years) and 20 chronic whiplash patients $(40 \pm 2.8$ years $)$.

The results of the different measurement tasks (see below) for each suspected malingering patient were judged by an experienced researcher (J.A.), who decided whether to classify the patient as suspected malingerer. Criteria used for inclusion were inconsistencies between subjective symptoms, and between symptoms and measurement task outcome performance, clinical history and results of the clinical tests. A malingering performance was determined as better or equal performance on more difficult tasks compared to easier ones. Subjects with phobic postural vertigo (PPV) were excluded based on sway for tandem walking and difficult stance tests (standing with eyes closed on foam) being normal [Querner et al., 2000]. Non-organic patient characteristics are shown in table 1.

The UVL patient data were obtained from a previously published follow-up study [Allum et al., 2003]. Some characteristic features of the UVL patients in comparison with healthy controls were recovery to normal for stance tasks 3 weeks after onset of acute symptoms. More difficult standing tasks, such as standing on foam with eyes open, or standing on 1 leg with eyes open were within normal limits after 3 months (defined as sway less than mean +2 SD of normal). Some gait tasks, such as walking $3 \mathrm{~m}$ while rotating the head, however, still showed abnormal sway patterns 3 months after onset. Overall, the patients were considered recovered 3 months after the acute onset of their balance deficit. 
The original acute status was based on the presence of spontaneous nystagmus, caloric response asymmetry greater than $55 \%$, and asymmetric vestibular ocular responses to $20 \% \mathrm{~s}^{2}$ whole body rotations about the vertical axis.

The whiplash patient data were also obtained from a previously published study [Sjöström et al., 2003]. The main goal in this study was to obtain a quantified screening battery for the balance deficits in chronic whiplash injury patients with clinical evidence of a trauma. Typical features for this group of patients were a greater sway while standing on 2 legs with eyes open on foam or on a normal surface compared to healthy controls. One-legged stance tasks showed a significantly lower duration in comparison to the controls. For the simple gait tasks, such as walking while rotating the head, whiplash patients showed less trunk sway.

Control subjects were randomly selected but age-matched out of an already existing database of normal controls [Hegeman et al., 2007]. On average, there were no height or weight differences between the sample populations. All normal subjects were free of orthopaedic, vestibular, or somatosensory disorders.

\section{Measurement System}

Trunk sway was measured with the Swaystar system (Balance International Innovations $\mathrm{GmbH}$, Switzerland) which consists of two angular velocity transducers mounted on the hardened part of an elasticised motorcycle belt, strapped to the lower back at the level of $\mathrm{L}_{2}-\mathrm{L}_{3}$. One transducer was oriented to measure angular velocity deviations in the roll plane (side-to-side) and one transducer was oriented in the pitch plane (fore-aft). Angular deviations were calculated online by using trapezoid integration of angular velocities sampled every $10 \mathrm{~ms}$.

\section{Measurement Tasks}

The battery of tasks used was derived from the Tinetti performance-oriented assessment of mobility [Tinetti, 1986] and the clinical test of sensory interaction in balance [Shumway-Cook and Horak, 1986]. It also features in several other studies [e.g. Allum and Adkin, 2003; Sjöström et al., 2003; Hegeman et al., 2007; Adkin et al., 2005]. Each patient was asked to complete the battery of 14 gait and stance tasks in order of increasing difficulty. All tasks were performed without shoes to avoid the effect of different shoe types affecting the data. Table 2a lists stance and gait tasks used for the analysis and table $2 b$ those not used as not all malingering patients appeared to be able to complete these tasks. For all stance tasks, the subject was asked to stand naturally for $20 \mathrm{~s}$, or until a near fall occurred, with the feet at shoulder width and arms hanging beside the body. A near fall was defined as a loss of balance requiring assistance from spotters standing behind the subject. The foam support surface used had a height of $10 \mathrm{~cm}$, a length of $204 \mathrm{~cm}$, a width of $44 \mathrm{~cm}$, and a density of $25 \mathrm{~kg} / \mathrm{m}^{3}$.

\section{Data Analysis}

The maximum peak-to-peak and 90\% excursions of angle and angular velocity in the pitch and roll directions were calculated for each task. To derive the individual $90 \%$ values a histogram of pitch and roll samples of each subject's trial was developed by dividing the peak-to-peak range into 40 bins. The $90 \%$ range was then established with the extreme $5 \%$ of values excluded in the histogram.
Table 2. Criteria and variables overview

a Analysed tasks, data used

\begin{tabular}{ll}
\hline s2eo & standing on 2 legs, eyes open $(20 \mathrm{~s})$ \\
s2ec & standing on 2 legs, eyes closed $(20 \mathrm{~s})$ \\
s2eof & standing on 2 legs, eyes open on foam $(20 \mathrm{~s})$ \\
s2ecf & standing on 2 legs, eyes closed on foam $(20 \mathrm{~s})$ \\
s1eo & standing on 1 leg, eyes open $(20 \mathrm{~s}$ or less $)$ \\
w8teo & walking 8 tandem steps, eyes open \\
w3mph & walking $3 \mathrm{~m}$, and pitching the head \\
w3mrh & walking $3 \mathrm{~m}$, and rotating the head \\
\hline
\end{tabular}

b Analysed tasks, data not used

\begin{tabular}{ll}
\hline slec & standing on 1 leg, eyes closed (20 s or less) \\
sleof & standing on 1 leg, eyes open on foam (20 s or less) \\
w8teof & walking 8 tandem steps, eyes open on foam \\
w3mec & walking $3 \mathrm{~m}$, eyes closed \\
Barriers & walking over 4 low barriers $24 \mathrm{~cm}$ high, spaced $1 \mathrm{~m}$ apart \\
Stairs & walking up and down 2 steps
\end{tabular}

c Criteria

\begin{tabular}{|c|c|}
\hline Crit. 1 & $(s 2 e o+s 2 e c)-(s 2 e o f+s 2 e c f)$ \\
\hline Crit. 2 & (w3mphRolVel - w3mhpPitVel) \\
\hline Crit. 3 & (w3mrhRolVel - w3mhrPitVel) \\
\hline Crit. 4 & (w8teoPitAng - w8teoRolAng) \\
\hline Crit. 5 & (w8teoPitVel - w8teoRolVel) \\
\hline Crit. 6 & $(\mathrm{~s} 2 \mathrm{ec}-\mathrm{s} 2 \mathrm{eo})$ \\
\hline Crit. 7 & (s2ecf - s2eof) \\
\hline Crit. 8 & (w8teo - s2ecf) \\
\hline Crit. 9 & (w8teo - sleo) \\
\hline Crit. 10 & $(s 2 e c f-s 2 e c)$ \\
\hline Crit. 11 & (s2eo) \\
\hline
\end{tabular}

Rol = Roll; Pit = Pitch; Vel = velocity Ang = angle.

\section{Criteria}

Based on previous literature (see Introduction) and our own observations [Allum and Carpenter, 2005], we developed 11 different criteria, which could suggest a malingering performance (table 2c) based on performance inconsistent with the task or task difficulty.

Criterion 1 compares the 2 relatively easy 2-legged stance tasks with 2 more difficult tasks standing on foam. This criterion is comparable with the criterion Cevette et al. [1995] and Gianoli et al. [2000] used in their studies with dynamic posturography. Criterion 2 examined whether trunk roll velocity was greater compared to the velocity in pitch direction during walking while pitching the head, whereas criterion 3 used the same comparison for the task of walking while rotating the head from side to side. Criterion 4 looked whether pitch angle was larger than trunk roll angle during walking 8 tandem steps, whereas criterion 5 used the same task to look whether pitch velocity was greater than roll velocity. Criterion 6 compares the difference in task performance 
between standing on 2 legs with eyes open and standing on 2 legs with eyes closed. Criterion 7 compares the difference between standing on 2 legs with eyes open on foam and standing on 2 legs with eyes closed on foam. Criterion 8 compares walking 8 tandem steps with eyes open and standing on 2 legs with eyes closed on foam. Criterion 9 compares standing on 1 leg with eyes open and walking 8 tandem steps. Criterion 10 compares standing on 2 legs with eyes closed on foam with measures for standing on 2 legs with eyes closed. Criterion 11 looks at standing on 2 legs with eyes open alone and is comparable to the criterion used by Goebel et al. [1997].

The criteria 1, 7, 8, 9, 10 and 11 were calculated for each variable separately; i.e. for roll angle, pitch angle, roll velocity and pitch velocity. The other criteria combined different variables.

\section{Statistical Analysis}

Independent $t$ tests were used to determine differences in the variables for each task and the criteria based on the variables between malingering patients and 1 of the other 3 groups. A 4-group ANOVA and post hoc Bonferroni analysis were performed as well. Significance was set at 0.05 .

Stepwise discriminant analysis was performed on all variables and criteria that were significantly different between at least 2 groups. A separate discriminant analysis was performed for the malingering versus control group, the malingering versus UVL group and the malingering versus whiplash group, using the significant variables and criteria determined from independent $t$ tests. Discriminant analysis including all 4 groups was performed as well. The input for this analysis was based on the significant outcomes of the ANOVA and post hoc Bonferroni tests. Stepwise discriminant analysis first carries out an ANOVA of each variable and then selects the variable with the highest $\mathrm{F}$ ratio for inclusion in the discriminant function. The correlation of the remaining variables (those that were not selected) to the variable selected to enter into the group of entered variables is then removed. In the next step, ANOVA is repeated on the remaining variables in order to determine the next most significant variable to enter the discriminant function. This stepwise procedure is repeated until all non-entered variables have $\mathrm{F}$ ratios less than a set threshold of $\mathrm{F}=3.84$. The set of variables entered into the discriminant function can then be used to classify all members of the specific groups to the appropriate population and to assign a new test subject to the appropriate population. A detailed description of this analysis is given elsewhere [Allum et al., 2001; Allum and Adkin, 2003].

\section{Results}

Overall, the malingering patients had a different performance on the different stance and gait tasks compared to the control, UVL and whiplash patient groups, which was characterized by larger trunk sway than is typical in patients with balance problems of organic origin. A typical record of a malingering patient during standing on foam with eyes closed is shown in figure 1a. The malingering patient has, in comparison with the other 3 subjects, greater sway in both the roll and pitch directions.
Because the $90 \%$ values gave a better differentiation between the different groups than the $100 \%$ range (peak-topeak) values, we will only report the results obtained with the $90 \%$ values.

\section{Stance Task Variables}

For the simplest task of standing on 2 legs on a firm surface with eyes open, roll angle and velocity were significantly increased between malingering and control patients. Pitch angle and pitch velocity were significantly increased between the malingering versus control and UVL patients $(\mathrm{p}<0.05)$. This was the variable which featured directly as a criteria (criteria 11, see table $2 \mathrm{c}$ ).

For standing on 2 legs on foam with eyes open, a significant increase was found between the malingering patients and the other 3 groups for the variables roll angle, roll velocity and pitch velocity. Pitch angle showed significant differences between the malingering versus control and UVL patients $(\mathrm{p}<0.05)$.

For the most difficult 2-legged stance task standing on foam with eyes closed (fig. 1b), a significant difference was found when comparing the malingering group with each one of the other 3 groups for all variables $(p<0.05)$. The task standing on 2 legs on a firm surface with eyes closed showed a significant difference between the malingering patients and the other 3 groups for all variables as well ( $\mathrm{p}<0.05)$.

For standing on 1 leg with eyes open, roll angle, pitch angle and pitch velocity were significantly increased between malingering patients and the other 3 groups. Roll velocity was significantly increased between the malingering and control groups $(\mathrm{p}<0.05)$.

\section{Gait Task Variables}

For walking 8 tandem steps with eyes open (fig. 1c), a significant increase was found between the malingering group and the other 3 groups for roll and pitch angle, as well as for pitch velocity $(\mathrm{p}<0.05)$. Roll velocity only showed a significant increase between the malingering group compared to the control group and whiplash group $(\mathrm{p}<0.05)$.

For walking $3 \mathrm{~m}$ while rotating the head, roll and pitch angle were significantly increased between the malingering patients versus controls and whiplash patients. Pitch velocity showed a significant difference between malingering and whiplash patients $(\mathrm{p}<0.05)$.

For walking $3 \mathrm{~m}$ while pitching the head, roll angle was significantly increased in the malingering group compared to controls and whiplash patients $(\mathrm{p}<0.05)$. 
Fig. 1. Balance performance. Trunk sway deviations (a) of 4 typical patients, representing the 4 groups during standing on 2 legs with eyes closed on a foam support surface. Note the larger ordinate scale for the malingering patient. b, c Population averages (and standard error of the mean) for trunk angle and velocity as measured for $90 \%$ ranges (see Methods) during standing on 2 legs with eyes closed on foam and while walking tandem steps. Significant differences based on a one-way ANOVA and post hoc Bonferroni tests are marked with a $\mathrm{p}<0.05$ control vs. malingering, ${ }^{b} \mathrm{p}<0.05$ malingering vs. UVL, and ${ }^{\mathrm{c}} \mathrm{p}<0.05$ malingering vs. whiplash.

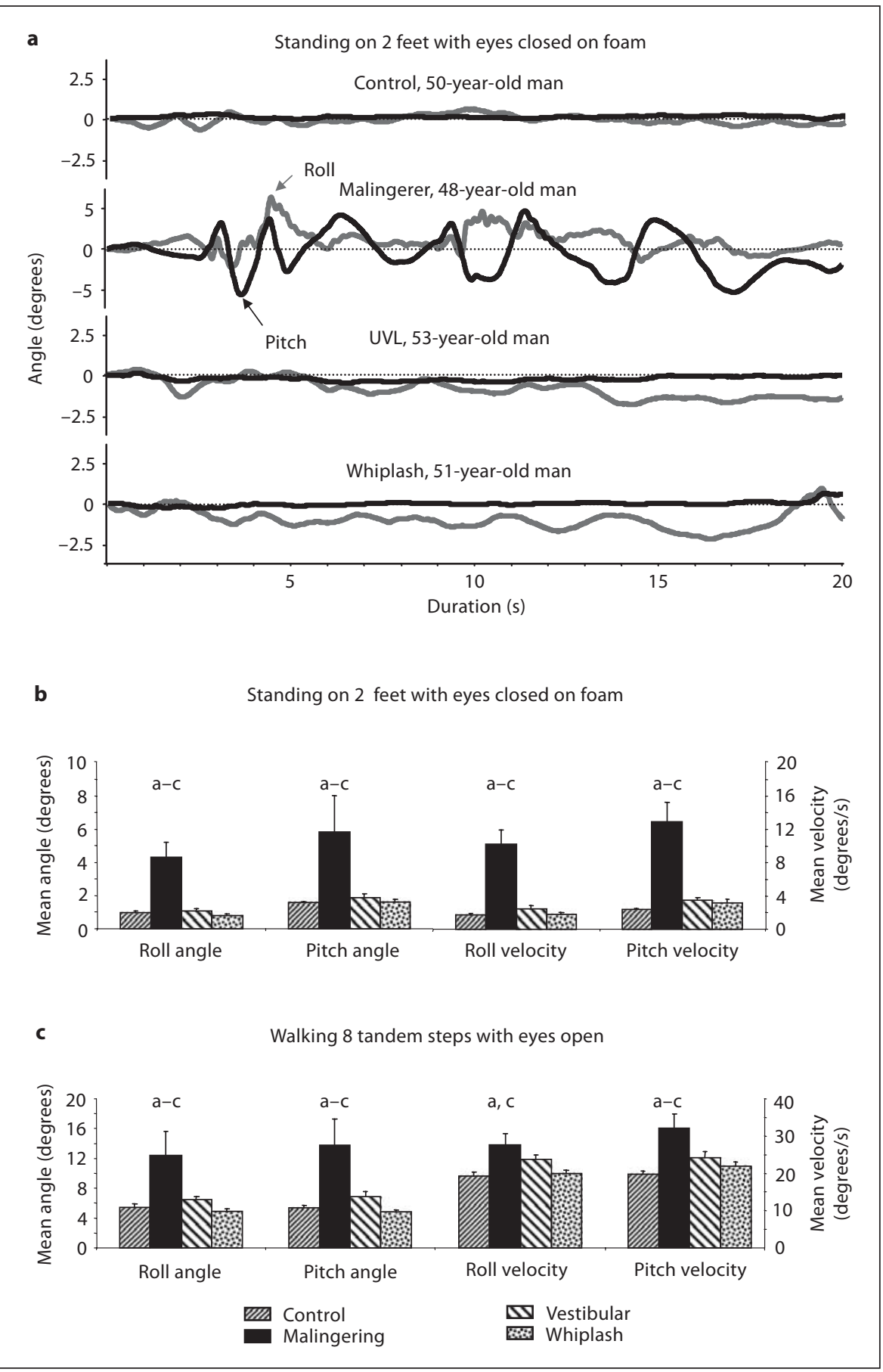

\section{Criteria}

Criterion 1, which compares standing on 2 legs on a firm surface with eyes open and closed, with standing on a foam surface with eyes open and closed, showed a significant increase between the malingering and the other 3 groups in all 4 variables $(\mathrm{p}<0.05)$.
Criterion 9, which calculates the difference between walking 8 tandem steps and standing on 1 leg with eyes open (fig. 2a), showed a significant decrease for the malingering group compared to the other 3 groups in roll and pitch velocity $(\mathrm{p}<0.05)$. 
Fig. 2. Criteria outcome. Population averages (and standard error of the mean) for criteria 9 (a) and 10 (b) are depicted (90\% ranges). Significant differences based on a one-way ANOVA and post hoc Bonferroni tests are marked with ${ }^{\mathrm{a}} \mathrm{p}<0.05$ control vs. malingering, ${ }^{b} \mathrm{p}<0.05$ malingering vs. $\mathrm{UVL}$, and ${ }^{\mathrm{c}} \mathrm{p}<0.05$ malingering vs. whiplash.

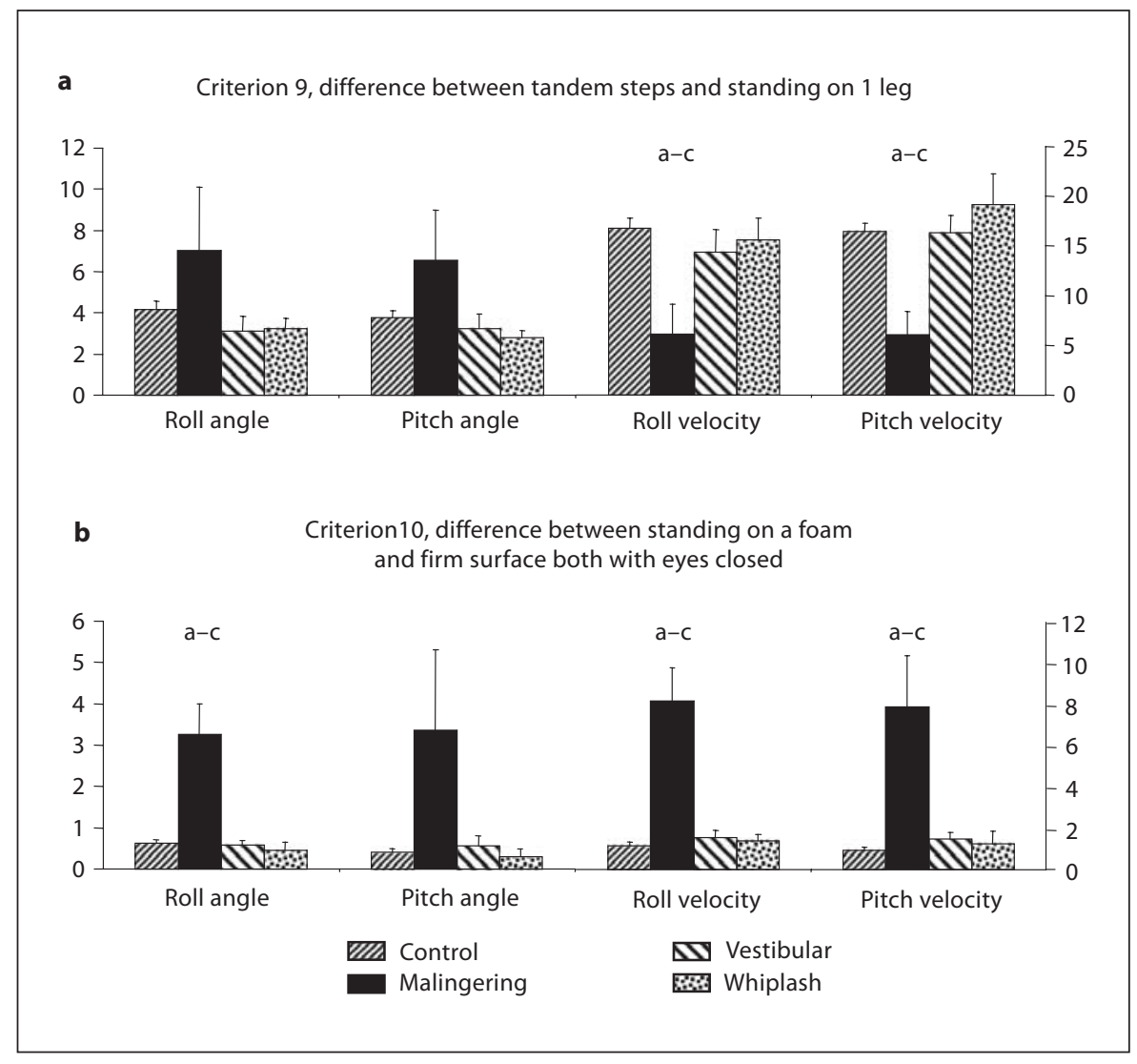

Criterion 10, which calculates the difference between standing on 2 legs with eyes closed on foam versus on a firm surface (fig. 2b), showed a significant increase for the malingering group compared to the other 3 groups in roll angle, roll velocity and pitch velocity. Pitch angle showed a significant increase between the malingering and control groups $(\mathrm{p}<0.05)$.

Criterion 11, which examines whether abnormally increased sway patterns are measured for standing on 2 legs with eyes open, showed a significant increase in roll angle and roll velocity for the malingering versus control groups. Pitch angle and pitch velocity were increased for the malingering group versus controls and UVL patients $(\mathrm{p}<0.05)$.

All remaining criteria not mentioned above did not show any significant differences between the malingering and 1 of the other 3 groups.

\section{Differentiation Based on 90\% Task Variables}

Figure 3 gives the classification results for the discriminant analysis for the 3 separate 2-group comparisons (fig. 3a) and the 4-group comparison (fig. 3b). Table 3a gives the details for each discriminant analysis. When differentiating the control group from the malingering group (fig. 3a), the overall classification was $96 \%$, all controls were correctly classified and $89 \%$ of the malingering patients were correctly classified. The differentiating variables are given in table $3 a$. The discriminant analysis between the malingering group and UVL group (fig. 3a) gave an overall correct classification of $76 \%$; $56 \%$ of the malingering patients were correctly classified and $95 \%$ of UVL patients were correctly classified (table $3 a$ for differentiating variables). The discriminant analysis between the malingering and whiplash group (fig. 3a) gave an overall classification of $84.2 \%$; all whiplash patients were correctly classified, and $67 \%$ of the malingering patients were correctly classified (table $3 a$ ).

The discriminant analysis between the 4 different groups (fig. 3b) gave an overall correct classification of $72 \%$; $94 \%$ of the controls, $67 \%$ of the malingerers, $50 \%$ of UVL and $60 \%$ of the whiplash patients were correctly classified (table $3 \mathrm{a}$ ). None of the subjects belonging to either the control, vestibular or whiplash groups were incorrectly classified as belonging to the malingering 
Fig. 3. Classification results. Discriminant plots, showing the differentiation obtained by the discriminant analysis between the patient groups, are illustrated. a, c The results of the 3 discriminant analyses between malingering versus controls, malingering versus UVL and malingering versus whiplash patients are provided based on $90 \%$ range variables (a) and $90 \%$ criteria (c). b, d The results of the discriminant analyses between the 4 groups based on the $90 \%$ variables (b) and $90 \%$ criteria (d) are depicted. For $\mathbf{b}$ and $\mathbf{d}$, only the first 2 out of 3 functions are used to plot the subjects.

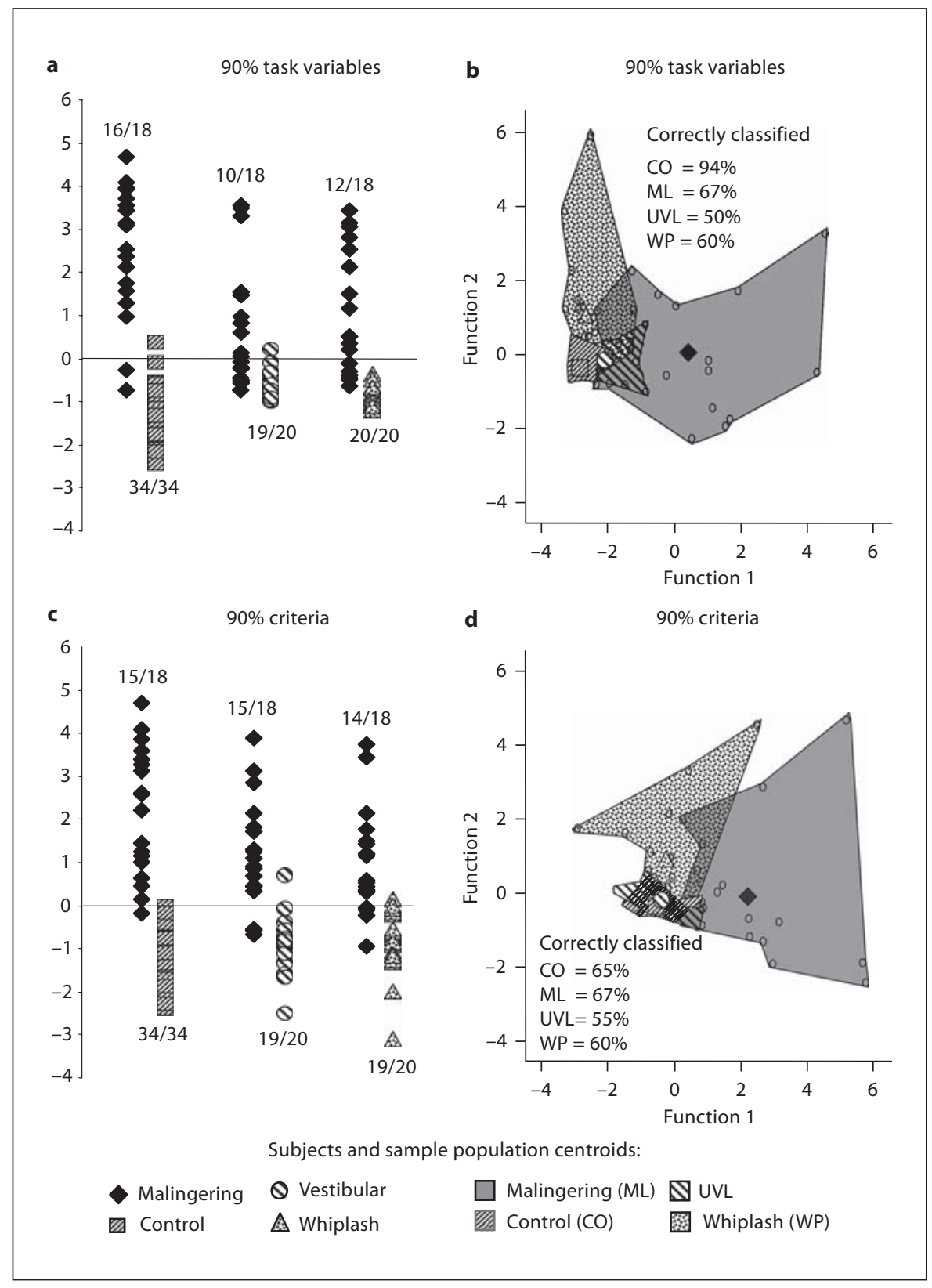

group. The classification function coefficients and Fisher's linear discriminant functions enabling classification of a new patient can be found in table $4 \mathrm{a}$.

\section{Differentiation Based on 90\% Criteria}

Figure $3 \mathrm{c}$ provides the classification results of the 3 comparisons separately, and figure $3 \mathrm{~d}$ of the 4-group comparison, based on the details given in table $3 \mathrm{~b}$. The discriminant analysis for the $90 \%$ criteria gave similar results to those of the $90 \%$ task variables. The discrimi- nant analysis for controls versus malingerers (fig. 3c) gave an overall classification of $94 \%$, thereby correctly classifying all controls and $83 \%$ of the malingerers (table $3 \mathrm{~b}$ ). The discriminant analysis between the malingering and UVL groups (fig. 3c) gave an overall classification of $90 \%$; $83 \%$ of the malingering patients and $95 \%$ of the UVL patients were correctly classified (table $3 \mathrm{~b}$ ). This was considerably better than for the $90 \%$ task variables. The discriminant analysis between the malingering group and whiplash group (fig. 3c) gave an overall correct classifica- 
Table 3. Outcome discriminant analysis

a Variables $90 \%$

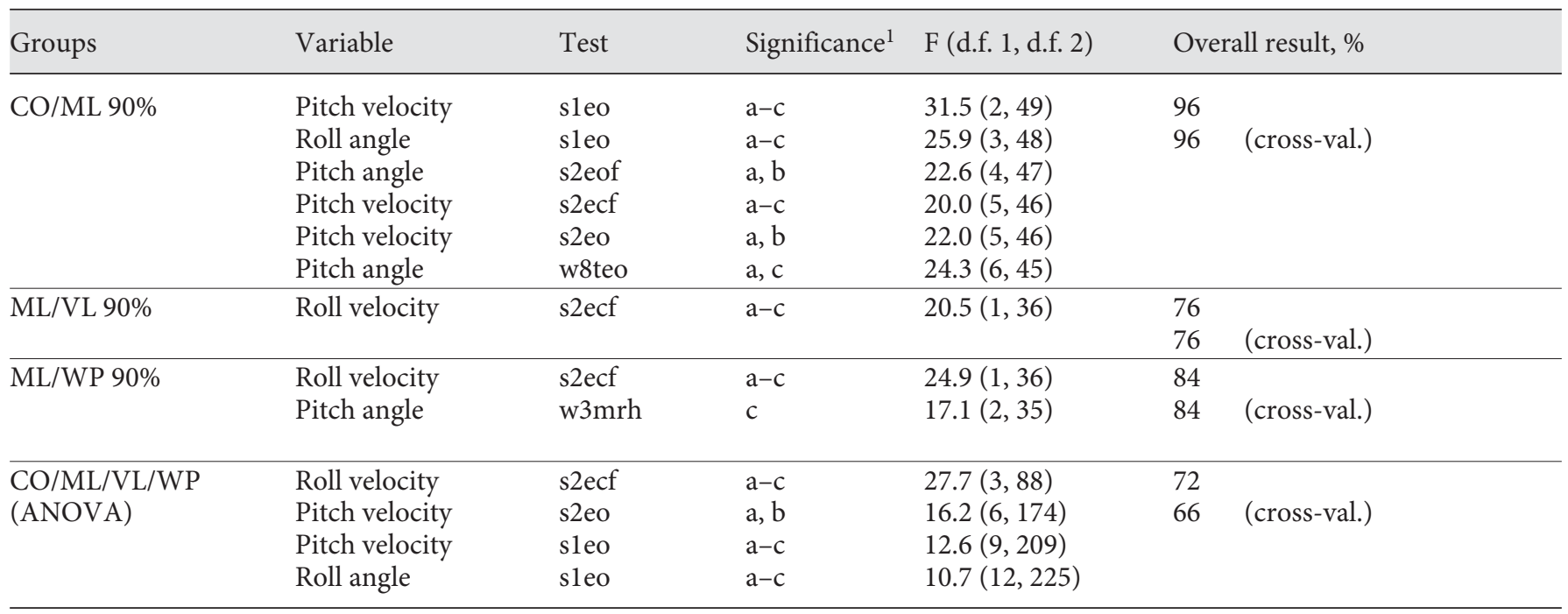

b Criteria $90 \%$

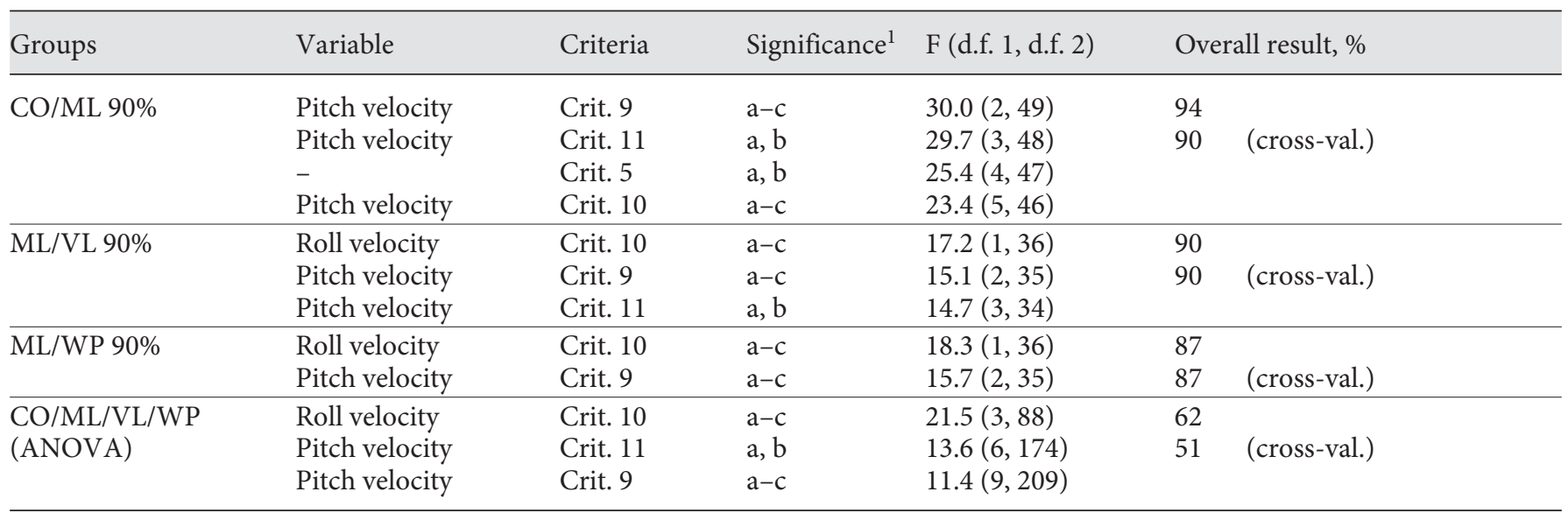

a: $\mathrm{p}<0.05$ CO vs. ML; b: $\mathrm{p}<0.05 \mathrm{ML}$ vs. VL; $\mathrm{c}$ : $\mathrm{p}<0.05 \mathrm{ML}$ vs. WP.

$\mathrm{CO}=$ Controls; $\mathrm{ML}=$ malingering; $\mathrm{VL}=$ vestibular loss; $\mathrm{WP}=$ whiplash patient; cross-val. $=$ cross-validated classification result. Each $F$ value had a $\mathrm{p}$ value $<0.05$.

${ }^{1}$ Significance for $\mathrm{t}$ tests, except for the 4-group comparison where significance values are given for ANOVA and Bonferroni tests.

tion of $87 \%$; $78 \%$ of the malingering patients and $95 \%$ of the whiplash patients were correctly classified (table $3 \mathrm{~b}$ ). This was slightly better than for the $90 \%$ task variables.

The discriminant analysis between the 4 different groups (fig. $3 \mathrm{~d}$ ) based on the selected criteria gave an overall classification of $62 \%$; $65 \%$ of the controls, $67 \%$ of the malingerers, $55 \%$ of the UVL patients and $60 \%$ of the whiplash patients were correctly classified. The same criteria were used as with the differentiation between malingering compared to UVL patients (table 3b). The Fish- er's linear discriminant function and the unstandardized canonical discriminant function coefficients enabling classification of a new patient can be found in table $4 \mathrm{~b}$.

\section{Discussion}

Our results showed clear differences in balance control during several stance and gait tasks between suspected malingerers and controls, UVL and whiplash patients. 
Table 4. Fisher's linear discriminant function (for the different subject groups) and unstandardized canonical discriminant function coefficients (for functions 1-3)

a Task variables $90 \%$

\begin{tabular}{|c|c|c|c|c|c|c|c|}
\hline & \multicolumn{4}{|c|}{ Subject group } & \multicolumn{3}{|c|}{ Function } \\
\hline & $\mathrm{CO}$ & ML & UVL & WP & 1 & 2 & 3 \\
\hline Standing on 1 leg EO, roll angle & -0.033 & 0.165 & 0.013 & -0.096 & 0.070 & -0.056 & 0.028 \\
\hline Standing on 1 leg EO, pitch velocity & 0.083 & 0.335 & 0.209 & 0.179 & 0.071 & 0.047 & 0.140 \\
\hline Standing on 2 legs on foam EC, roll velocity & 0.060 & 0.614 & 0.067 & -0.064 & 0.196 & -0.094 & -0.172 \\
\hline Standing on 2 legs EO, pitch velocity & 0.642 & 0.715 & 0.642 & 1.722 & -0.080 & 0.795 & -0.195 \\
\hline Constant & -1.934 & -9.000 & -2.756 & -4.240 & -1.342 & -1.290 & -0.122 \\
\hline
\end{tabular}

b Criteria $90 \%$

\begin{tabular}{|c|c|c|c|c|c|c|c|}
\hline & \multicolumn{4}{|c|}{ Subject group } & \multicolumn{3}{|c|}{ Function } \\
\hline & $\mathrm{CO}$ & ML & UVL & WP & 1 & 2 & 3 \\
\hline Criteria 9, Pitch velocity & 0.219 & 0.029 & 0.212 & 0.220 & -0.070 & 0.021 & 0.098 \\
\hline Criteria 10 , Roll velocity & 0.075 & 0.684 & 0.109 & 0.034 & 0.226 & -0.102 & 0.191 \\
\hline Criteria 11 , Pitch velocity & 0.252 & 1.180 & 0.353 & 1.134 & 0.281 & 0.683 & -0.185 \\
\hline Constant & -3.379 & -5.958 & -3.451 & -5.128 & -0.091 & -1.348 & -1.623 \\
\hline
\end{tabular}

$\mathrm{EO}=$ Eyes open $\mathrm{EC}=$ eyes closed $\mathrm{CO}=$ Controls; $\mathrm{ML}=$ malingering; $\mathrm{UVL}=$ unilateral vestibular loss; $\mathrm{WP}=$ whiplash patient.

When combining the best discriminant analysis outcomes, an overall correct classification of $86 \%$ was obtained. The exaggerated responses of the malingerers can be explained by the expected financial gain under the Swiss Invalidity Insurance programme. Costs per case in Switzerland are the highest in Europe [Committee of European Assurances, 2004].

Trunk pitch velocity featured in several of the variables and criteria from stance tasks used to distinguish malingerers from other patients. Derived pitch angle was used by other authors [Goebel et al., 1997; Hamid et al., 1990; Mallison et al., 2005] employing sway-referenced dynamic posturography to identify malingerers. We found that roll velocity was particularly useful for separating malingerers and whiplash patients. More importantly, we demonstrated that using differences between a gait task (walking tandem steps) and a stance composite of that task (standing on 1 leg with eyes open) improved classification accuracy over that obtained for stance variables alone. The variable employed to focus on these differences was pitch velocity. It is indicative of malingering if pitch velocity is greater than roll velocity during tandem walking because usually the greatest motion is in the roll direction for organic disorders of balance [Allum and Carpenter, 2005; Allum and Adkin, 2003]. Generally though, gait tasks failed to provide improved classification accuracy over stance tasks even though there were significant differences between the malingerers and controls as well as whiplash injury patients for gait tasks. Only the walking $3 \mathrm{~m}$ while rotating the head task entered into the discrimination between malingerers and whiplash patients. Whiplash injury patients are characterized by low values of trunk sway variables for this test [Sjöström et al., 2003].

Standing on 2 legs with eyes open is generally associated with the smallest amount of sway even for patients with organic balance deficits [Allum and Carpenter, 2005; Allum and Adkin, 2003]. Thus, poor performance on this test is immediately suspicious as others have found.

Goebel et al. [1997] tested 3 different groups, consisting of normal subjects, patients with balance disorders and normal subjects instructed to feign a balance performance. One abnormal sway pattern for the latter group was the increase in sway during standing on 2 legs with eyes open. Several authors [Hamid et al., 1990; Mallin- 
son et al., 2005; Endo et al., 2008] found that the same task provided significantly different measures for their malingering groups. In the current study, malingering patients had a significant increase in sway for this task, too. Pitch velocity for this task emerged as a discriminating variable for 3 out of 4 comparisons between the different patient groups, confirming our assumption that every patient should be able to perform this task within normal limits. Some caution is needed, because Allum et al. [2003] found a significant increase with this task for whiplash patients compared to controls, which might explain why this test was not useful for differentiating malingerers from whiplash patients in our discriminant analysis.

Cevette et al. [1995] indicated that a relatively better performance on difficult tasks compared to easier tasks is a typical feature for malingering patients. Gianoli et al. [2000] and Rey-Martinez et al. [2007] provided support for this assumption. Krempl and Dobie [1998], who compared the Cevette score, the Goebel criteria and blinded subjective clinical scoring for the ability to correctly differentiate the malingering patients, did not find the above-mentioned Cevette criterion helpful for differentiating the malingering group. One of their main conclusions was that blinded clinical scoring gave comparable differentiation results to the other 2 test methods. To determine whether the Cevette criterion was able to differentiate the malingering patients, we integrated this concept into a number of criteria (1, 6, 7 and 10 , see table $2 c)$ comparing a difficult and easy task. Although the criteria were often significantly different between the malingering group and the other 3 groups, we did not find any statistically significant evidence that the more difficult tasks resulted in better performance, compared to the easier tasks. Instead higher differences between the difficult task (foam) and easy task (firm surface) for the trunk pitch and roll velocity in malingerers versus controls and other patient groups were observed (fig. 2b). Our results showed that a greater-than-expected difference between difficult and easy tasks is suggestive of a malingering performance. The difference between standing with eyes closed on foam and on a firm surface emerged as a significant variable in the discriminating function for all analyses.

None of the control, UVL or whiplash patients were falsely classified as belonging to the malingering group when a 4-group differentiation was performed, either based on the task variables alone or the criteria. It appears that the discriminant analysis gave very specific results in differentiating malingerers, resulting in no false-posi- tive results. This is desirable from the medical point of view, because a clinician does not want to face the risk of classifying a true organic patient as being malingering. For this reason, a high specificity, as we obtained, is required. In fact, many of the falsely classified subjects were healthy controls classified as UVL subjects and vice versa. Such a poor classification was expected, a priori, because UVL subjects 3 months after onset of their symptoms are generally symptom free [Allum and Adkin, 2003].

In the current work, we have considered all patients in the malingerer group to be malingerers. Three of the patients had a pathological canal paresis indicating a peripheral vestibular loss and should strictly be assigned to a group of aggravating patients. Future work will need to consider differences between pure malingerers and those aggravating an organic deficit. Another aspect which will need to be considered is the differences between malingerers, aggravations and those patients with PPV. PPV patients have normal results on difficult stance tasks, but abnormal results for the easier balance tasks [Querner et al., 2000] unless the task is expected to be difficult [Holmberg et al., 2009]. We excluded PPV patients based on the criteria by Querner et al. [2000]. However, when both stance and gait tasks are used for differentiation of PPV from normal control, the question arises concerning which tasks are the most difficult - stance or gait tasks or a combination of both - to be used for identification of PPV patients.

A remaining question arises, whether malingerers who are aware of the above-mentioned criteria used to identify a malingering performance would still be detected as being malingering. Morgan et al. [2002] looked whether dynamic posturography was able to detect informed malingerers, based on the scoring method of Cevette et al. [1995] and blinded subjective clinical scoring. They investigated 2 groups of healthy subjects who both were asked to simulate a balance deficit. The first group, however, was informed beforehand about dynamic posturography and that a patient with a balance disorder tends to do poorly only when the test becomes more difficult. Despite additional information, the patients did not improve their ability to feign a balance disorder. Comparable classification results were found between both groups, with either the scoring method of Cevette et al. [1995] or subjective blinded clinical scoring.

Our work has focused on providing criteria based on trunk sway measured during stance and gait tasks to differentiate malingerers from controls, UVL and whip- 
lash patients and thereby provide an objective method for identifying malingering patients. Future research will need to determine how these techniques work in prospectively identifying patients who are malingering.

\section{Acknowledgments}

This project was supported by a grant from the Swiss National Research Foundation (No. 320000-117950) to J.H.J. Allum and a grant from SUVA (Swiss Accident Insurance Institution). Jaap Vonk was supported by the European Student Exchange Program (StudEx).

\section{References}

Adkin AL, Bloem BR, Allum JHJ: Trunk sway measurements during stance and gait tasks in Parkinson's disease. Gait Posture 2005;22: 240-249.

Allum JHJ, Adkin AL: Improvements in trunk sway observed for stance and gait tasks during recovery from an acute unilateral peripheral vestibular deficit. Audiol Neurootol 2003;8:286-302.

-Allum JHJ, Bloem BR, Carpenter MG, Honegger F: Differential diagnosis of propioceptive and vestibular deficits using dynamic support-surface posturography. Gait Posture 2001;14:217-226.

-Allum JHJ, Carpenter MG: A speedy solution for balance and gait analysis: angular velocity measured at the centre of body mass. Curr Opin Neurol 2005;18:15-21.

-Allum JHJ, Huwiler M, Honegger F: Identifying cases of non-organic vertigo using dynamic posturography. Gait Posture 1996;4:52-61.

Allum JHJ, Shepard NT: An overview of the clinical use of dynamic posturography in the differential diagnosis of balance disorders. J Vestib Res 1999;9:223-252.

Allum JHJ, Zamani F, Adkin AL, Ernst A: Differences between trunk sway characteristics on a foam support surface and on the Equitest ${ }^{\circledR}$ ankle-sway-referenced support surface. Gait Posture 2002;16:264-270.

Binder LM, Rohling ML: Money matters: a metaanalytic review of the effects of financial incentives on recovery after closed-head injury. Am J Psychiatry 1996;153:7-10.

-Cevette MJ, Puetz B, Marion MS, Wertz ML, Muenter MD: Aphysiologic performance on dynamic posturography. Otolaryngol Head Neck Surg 1995;112:676-688.

-Colebatch JG, Halmagyi GM, Skuse NF: Myogenic potentials generated by click-evoked vestibulocollic reflex. J Neurol Neurosurg Psychiatry 1994;57:190-197.
Committee of European Assurances (CEA): Frequency of deficits and case costs after minor damage to the cervical-spinal column. CERE DOC 2004.

Endo K, Suzuki H, Yamamoto K: Consciously postural sway and cervical vertigo after whiplash injury. Spine 2008;33:539-542.

Gianoli G, McWilliams S, Soileau J, Belafsky P: Posturographic performance in patients with the potential for secondary gain. Otolaryngol Head Neck Surg 2000;122:11-18.

Goebel JA, Sataloff RT, Hanson JM, Nashner LM, Hirshout DS, Sokolow CC: Posturographic evidence of nonorganic sway patterns in normal subjects, patients, and suspected malingerers. Otolaryngagol Head Neck Surg 1997;117:293-232.

Hain TC: Treatment of vertigo. Neurologist 1995;1:125-133.

Hamid MA: New tests of vestibular function; in Meyers EN, et al (eds): Advances in Otolaryngology-Head and Neck Surgery. Chicago, Year Book Medical Publishers, 1990, vol 5, pp 15-38.

Hegeman J, Shapkova EY, Honegger F, Allum JHJ: Effect of age and height on trunk sway during stance and gait. J Vestib Res 2007;17: 75-87.

Holmberg J, Tjernström F, Karlberg M, Fransson PA, Magnusson M: Reduced postural differences between phobic postural vertigo patients and healthy subjects during a postural threat. J Neurol 2009;256:1258-1262.

Horlings CGC, Küng UM, Bloem BR, Honegger F, Van Alfen N, Van Engelen BGM, Allum JHJ: Identifying deficits in balance control following vestibular or proprioceptive loss using posturographic analysis of stance tasks. Clin Neurophysiol 2008;119:23382346.

Johansson R, Magnusson M, Fransson PA, Karlberg M: Discrimination between patients with acoustic neuroma and with peripheral vestibular lesion by human posture dynamics. Acta Otolaryngol 1994;114:479-483.
Karlberg M, Johansson R, Magnusson M, Fransson PA: Dizziness of suspected cervical origin distinguished by posturographic assessment of human postural dynamics. J Vestib Res 1996;6:37-47.

Krempl GA, Dobie RA: Evaluation of posturography in the detection of malingering subjects. Am J Otol 1998;19:619-627.

Mallison AI, Longridge NS: A new set of criteria for evaluating malingering in work-related vestibular injury. Otol Neurotol 2005;26: 686-690.

Morgan SS, Beck WG, Dobie RA: Can posturography identify informed malingerers? Otol Neurotol 2002;23:214-217.

-Nedzelski JM, Barber HO, McIlmoyl L: Diagnosis in a dizziness unit. J Otolaryngol 1986; 15 : 101-104.

Pearce JMS: The myth of chronic whiplash injury. Spinal Cord 1999;27:741-748.

- Querner V, Krafczyk S, Dieterich M, Brandt T: Patients with somatoform phobic postural vertigo: the more difficult the balance task, the better the balance performance. Neurosc Lett 2000;285:21-24.

- Rey-Martinez J, Rama-Lopez J, Pérez-Fernandez $\mathrm{N}$ : Posturographic examination of malingering patients. Acta Otorrinolaringol Esp 2007;58:202-207.

-Shumway-Cook A, Horak FB: Assessing the influence of sensory interaction on balance. Phys Ther 1986;66:1548-1550.

-Sjöström H, Allum JHJ, Carpenter MG, Adkin AL, Honegger F, Ettlin T: Trunk sway measurers of postural stability during clinical balance tests in patients with chronic whiplash injury symptoms. Spine 2003;28:17251734.

Tinetti ME: Performance-oriented assessment of mobility problems in elderly patients. J Am Geriatr Soc 1986;34:119-126.

-Weber PC, Cass SP: Clinical assessment of postural stability. Am J Otol 1993;14:566-569. 